\title{
On Faster Convergence of the Bisection Method for Certain Triangles
}

\author{
By Martin Stynes
}

\begin{abstract}
Let $\triangle A B C$ be a triangle with vertices $A, B$ and $C$. It is "bisected" as follows: choose a/the longest side (say $A B$ ) of $\triangle A B C$, let $D$ be the midpoint of $A B$, then replace $\triangle A B C$ by two triangles, $\triangle A D C$ and $\triangle D B C$.

Let $\Delta_{01}$ be a given triangle. Bisect $\Delta_{01}$ into two triangles $\Delta_{11}, \Delta_{12}$. Next, bisect each $\Delta_{1 i}, i=1,2$, forming four new triangles $\Delta_{2}, i=1,2,3,4$. Continue thus, forming an infinite sequence $T_{j}, j=0,1,2, \ldots$, of sets of triangles, where $T_{j}=\left\{\Delta_{j i}: \quad 1 \leqslant i \leqslant 2^{j}\right\}$. It is known that the mesh of $T_{j}$ tends to zero as $j \rightarrow \infty$. It is shown here that if $\Delta_{01}$ satisfies any of four certain properties, the rate of convergence of the mesh to zero is much faster than that predicted by the general case.
\end{abstract}

1. Introduction. Let $\triangle A B C$ be a triangle with vertices $A, B$ and $C$. We define the procedure for "bisecting" $\triangle A B C$ as follows: choose a/the longest side (say $A B$ ) of $\triangle A B C$, let $D$ be the midpoint of $A B$, then divide $\triangle A B C$ into the two triangles $\triangle A D C$ and $\triangle D B C$.

Let $\Delta_{01}$ be a given triangle. Bisect $\Delta_{01}$ into two triangles $\Delta_{11}$ and $\Delta_{12}$. Next, bisect each $\Delta_{1 i}, i=1,2$, forming four new triangles $\Delta_{2 i}, i=1,2,3,4$. Set $T_{j}=$ $\left\{\Delta_{j i}: 1 \leqslant i \leqslant 2^{j}\right\}, j=0,1,2, \ldots$, so $T_{j}$ is a set of $2^{j}$ triangles. Define $m_{j}$, the mesh of $T_{j}$, to be the length of the longest side among the sides of the triangles in $T_{j}$. Clearly $0<m_{j+1} \leqslant m_{j}$ for all $j \geqslant 0$. It is shown implicitly in [3] that in fact $m_{j} \rightarrow 0$ as $j \rightarrow \infty$. Thus, this bisection method is useful in finite element methods for approximating solutions of differential equations (see e.g. [1]). A modification of such a bisection method can be used in computing the topological degree of a mapping from $R^{3}$ to $R^{3}$ ([4], [5]).

In [2] an explicit bound is obtained for the rate of convergence of $m_{j}: m_{j} \leqslant$ $(\sqrt{3} / 2)^{[j / 2]} m_{0}$, where $[x]$ denotes the integer part of $x$. In [2] it is also mentioned that computer experiments indicate that in many cases this bound is unrealistically high; this prompted the present results. We show that if $\Delta_{01}$ belongs to any one of four sets of equivalence classes of triangles, then we have the substantially improved bounds of Corollaries 1 and 2 below. Much of the notation used is taken from [3].

2. Results.

Definition. Given three positive numbers $\rho, \sigma, \tau$ such that $\rho+\sigma+\tau=\pi$, define $(\rho, \sigma, \tau)$ to be the set of all triangles whose interior angles are $\rho, \sigma, \tau$.

Received February 14, 1978.

AMS (MOS) subject classifications (1970). Primary 50B30, 50B15; Secondary 41 A63, $65 \mathrm{~N} 30,55 \mathrm{C} 25$. 
With this notation we divide the set of all triangles into similarity classes. Note that $(\rho, \sigma, \tau)=(\sigma, \rho, \tau)$ etc.

Definition. Given a triangle $\Delta$, define $d(\Delta)$, the diameter of $\Delta$, to be the length of the longest side of $\Delta$.

Definition. Given a similarity class $(\rho, \sigma, \tau)$ choose any triangle $\Delta \in(\rho, \sigma, \tau)$ and join the midpoint of its longest side to the opposite vertex. The new side ratio $r$ of $(\rho, \sigma, \tau)$ is defined to be the length of this new side divided by $d(\Delta)$.

Remark. The new side ratio is well-defined since it does not depend on the particular $\Delta$ chosen in $(\rho, \sigma, \tau)$. By Lemma 5.2(i) of [4] we have $0<r \leqslant \sqrt{3} / 2$ always.

Definition. Using the notation of the introduction, an iteration of the bisection method applied to $\Delta_{00}$ is defined to be the progression from $T_{j}$ to $T_{j+1}$ for any $j \geqslant 0$. A cycle of the bisection method is defined to be two successive iterations, i.e., the progression from $T_{j}$ to $T_{j+2}$ for any $j \geqslant 0$.

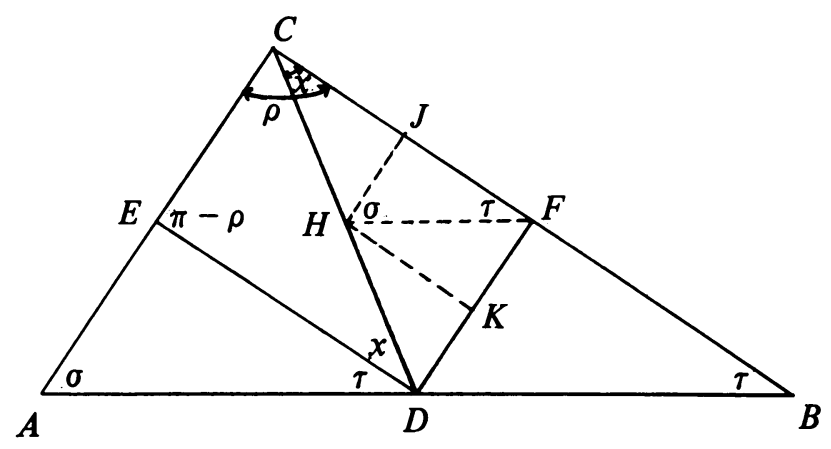

FigURE 1

In Figure $1, \triangle A B C \in(\rho, \sigma, \tau)$ and we have taken $\tau \leqslant \sigma \leqslant \rho$. Thus, $A C \leqslant B C$ $\leqslant A B$ (where ' $A C$ ' denotes 'length of $A C$ ' etc.). $D, E, F$ are the midpoints of $A B$, $A C, B C$, respectively. Under the additional hypothesis that $x+\tau \geqslant \max \{\sigma, \rho-x\}$ and $\pi-\rho \geqslant \rho-x$, bisections will take place exactly as in Figure 1 (see [3]).

Notation. To indicate that $\Delta_{1} \in\left(\alpha_{1}, \beta_{1}, \gamma_{1}\right)$ yields $\Delta_{2} \in\left(\alpha_{2}, \beta_{2}, \gamma_{2}\right)$ and $\Delta_{3}$ $\in\left(\alpha_{3}, \beta_{3}, \gamma_{3}\right)$ when bisected, we shall draw a pair of arrows emanating from the triple $\left(\alpha_{1}, \beta_{1}, \gamma_{1}\right)$ with one entering each of the triples $\left(\alpha_{2}, \beta_{2}, \gamma_{2}\right)$ and $\left(\alpha_{3}, \beta_{3}, \gamma_{3}\right)$.

We quote the following result from [3]; it uses the notation of Figure 1.

Lemma 1 [3, LemMa 4]. If $\tau \leqslant \sigma \leqslant \rho, x+\tau \geqslant \max \{\sigma, \rho-x\}$, and $\pi-\rho \geqslant$ $\rho-x$, then we must have

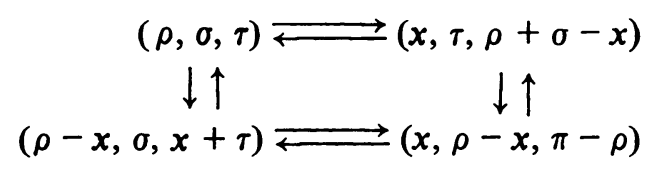

Proof. See [3].

Thus, after one cycle of the bisection method applied to $\triangle A B C \in(\rho, \sigma, \tau)$, we have two triangles from $(\rho, \sigma, \tau)(\triangle A D E, \triangle D B F$ above) and two triangles from $(x, \rho-x, \pi-\rho)(\triangle C E D, \triangle C F D$ above $)$. Now $d(A D E)=d(D B F)=d(A B C) / 2$. Also, 
$d(C E D)=d(C F D)=C D$ (since $\pi-\rho \geqslant \rho-x$ by hypothesis, and $\rho-x \geqslant x$ from $[3])$, i.e., $d(C E D)=d(C F D)=r d(A B C)$, where $r$ is the new side ratio of $(\rho, \sigma, \tau)$.

Similarly, after one cycle of the bisection method applied to $\triangle C F D \in(x, \rho-x$, $\pi-\rho)$, we obtain $\triangle H J F, \triangle H K F \in(\rho, \sigma, \tau)$ and $\triangle C J H, \triangle H K D \in(x, \rho-x, \pi-\rho)$. Here

$$
d(C J H)=d(H K D)=d(C F D) / 2,
$$

and

$$
d(H J F)=d(H K F)=H F=A B / 4=C D / 4 r=d(C F D) / 4 r .
$$

ThEOREM 1. Assume that $\tau \leqslant \sigma \leqslant \rho, x+\tau \geqslant \max \{\sigma, \rho-x\}$, and $\pi-\rho \geqslant$ $\rho-x$. Then for $n \geqslant 1$, after $n$ cycles of the bisection method applied to

(i) $\Delta \in(\rho, \sigma, \tau)$, we have $2^{2 n-1}$ triangles in $(\rho, \sigma, \tau)$ each with diameter $d(\Delta) / 2^{n}$ and $2^{2 n-1}$ triangles in $(x, \rho-x, \pi-\rho)$ each with diameter $d(\Delta) r / 2^{n-1}$;

(ii) $\Delta^{\prime} \in(x, \rho-x, \pi-\rho)$, we have $2^{2 n-1}$ triangles in $(x, \rho-x, \pi-\rho)$ each with diameter $d\left(\Delta^{\prime}\right) / 2^{n}$ and $2^{2 n-1}$ triangles in $(\rho, \sigma, \tau)$ each with diameter $d\left(\Delta^{\prime}\right) / 2^{n+1} r$.

Here $r$ is the new side ratio of $(\rho, \sigma, \tau)$.

Proof. We use induction on $n$. The case $n=1$ is proven in the remarks following Lemma 1.

Fix $k>1$. Assume that the theorem is true for $1 \leqslant n<k$. We prove it true for $n=k$.

First, part (i). After one cycle of the bisection method applied to $\Delta$, we have $\Delta_{1}, \Delta_{2} \in(\rho, \sigma, \tau)$ with $d\left(\Delta_{1}\right)=d\left(\Delta_{2}\right)=d(\Delta) / 2$, and $\Delta_{3}, \Delta_{4} \in(x, \rho-x, \pi-\rho)$ with $d\left(\Delta_{3}\right)=d\left(\Delta_{4}\right)=r d(\Delta)$. Applying a further $k-1$ cycles to each of these four triangles, we obtain from $\Delta_{1}$ and $\Delta_{2}$ by the inductive hypothesis $2^{2 k-2}$ triangles in $(\rho, \sigma, \tau)$ each with diameter $d\left(\Delta_{1}\right) / 2^{k-1}=d(\Delta) / 2^{k}$, and $2^{2 k-2}$ triangles in $(x, \rho-x$, $\pi-\rho$ ) each with diameter $r d\left(\Delta_{1}\right) / 2^{k-2}=r d(\Delta) / 2^{k-1}$; from $\Delta_{3}$ to $\Delta_{4}$ we get $2^{2 k-2}$ triangles in $(x, \rho-x, \pi-\rho)$ each with diameter $d\left(\Delta_{3}\right) / 2^{k-1}=r d(\Delta) / 2^{k-1}$ and $2^{2 k-2}$ triangles in $(\rho, \sigma, \tau)$ each with diameter $d\left(\Delta_{3}\right) / r 2^{k}=d(\Delta) / 2^{k}$. Adding totals of identical triangles shows that (i) holds for $n=k$.

By an analogous argument (ii) holds for $n=k$. This completes the proof.

Corollary 1. Suppose $\tau \leqslant \sigma \leqslant \rho, x+\tau \geqslant \max \{\sigma, \rho-x\}$, and $\pi-\rho \geqslant$ $\rho-x$. Then in the notation of the introduction

(i) If $\Delta_{01} \in(\rho, \sigma, \tau)$, then $m_{j} \leqslant \max \{r, 1 / 2\}(1 / 2)^{[j / 2]-1} d\left(\Delta_{01}\right)$ for $j \geqslant 1$, with equality for even $j$;

(ii) if $\Delta_{01} \in(x, \rho-x, \pi-\rho)$, then $m_{j} \leqslant \max \{1 / 2 r, 1\}(1 / 2)^{[j / 2]} d\left(\Delta_{01}\right)$ for $j \geqslant 1$, with equality for even $j$.

Proof. Immediate from Theorem 1.

Remark. In practice the conditions of Theorem 1 are more easily checked if expressed in terms of the lengths of sides of triangles. Using the notation of Figure 1,

$\tau \leqslant \sigma \leqslant \rho$ is equivalent to $A C \leqslant B C \leqslant A B$,

$x+\tau \geqslant \max \{\sigma, \rho-x\}$ is equivalent to $A C \geqslant \max \{A B / 2, C D\}$,

$\pi-\rho \geqslant \rho-x$ is equivalent to $C D \geqslant B C / 2$.

Thus, knowing the lengths of $A C, B C, A B$ and $C D$ one can immediately decide whether 
or not $\triangle A B C \in(\rho, \sigma, \tau)$ satisfies the conditions of Theorem 1. Note that these inequalities and $[4$, Lemma $5.2(i)]$ give $1 / 4 \leqslant r \leqslant \sqrt{3} / 2$.

Given a triangle such as $\triangle C F D$ with $C D \geqslant C F \geqslant D F$, to decide whether or not $\triangle C F D$ $\in(x, \rho-x, \pi-\rho)$ for some $(\rho, \sigma, \tau)$ where the various angles satisfy the conditions of Theorem 1 , bisect $C D$ at $H$ and $C F$ at $J$, then check (as above for $\triangle A B C$ ) whether or not $\Delta H J F \in(\rho, \sigma, \tau)$ satsifies the condtions of Theorem 1 with $H F \geqslant F J \geqslant J H$.

We now give a theorem similar to Theorem 1 which deals with the other two similarity classes mentioned in Lemma 1.

Definition. The smaller sides ratio $s$ of a similarity class $(\rho, \sigma, \tau)$ is obtained by choosing any $\triangle A B C \in(\rho, \sigma, \tau)$ with $A B \geqslant B C \geqslant A C$, then setting $s=B C / A C$.

Theorem 2. Assume that $\tau \leqslant \sigma \leqslant \rho, x+\tau \geqslant \max \{\sigma, \rho-x\}$, and $\pi-\rho \geqslant$ $\rho-x$. Then for $n \geqslant 1$, after $n$ cycles of the bisection method applied to

(i) $\Delta \in(\rho-x, \sigma, x+\tau)$, we have $2^{2 n-1}$ triangles in $(\rho-x, \sigma, x+\tau)$ each with diameter $d(\Delta) / 2^{n}$ and $2^{2 n-1}$ triangles in $(x, \tau, \rho+\sigma-x)$ each with diameter $s d(\Delta) / 2^{n}$ :

(ii) $\Delta^{\prime} \in(x, \tau, \rho+\sigma-x)$, we have $2^{2 n-1}$ triangles in $(x, \tau, \rho+\sigma-x)$ each with diameter $d\left(\Delta^{\prime}\right) / 2^{n}$ and $2^{2 n-1}$ triangles in $(\rho-x, \sigma, x+\tau)$ earh with diameter $d\left(\Delta^{\prime}\right) / 2^{n} s$.

Here $s$ is the smaller sides ratio of $(\rho, \sigma, \tau)$.

Proof. Analogous to that of Theorem 1.

Corollary 2. Suppose $\tau \leqslant \sigma \leqslant \rho, x+\tau \geqslant \max \{\sigma, \rho-x\}$, and $\pi-\rho \geqslant \rho-x$. Then in the notation of the introduction

(i) if $\Delta_{01} \in(\rho-x, \sigma, x+\tau)$, then $m_{j} \leqslant s(1 / 2)^{[j / 2]} d\left(\Delta_{01}\right)$ for $j \geqslant 1$, with equality for even $j$;

(ii) if $\Delta_{01} \in(x, \tau, \rho+\sigma-x)$, then $m_{j} \leqslant(1 / 2)^{[j / 2]} d\left(\Delta_{01}\right)$ for $j \geqslant 1$, with equality for even $j$.

Note that since we are assuming that $A C \geqslant \max \{A B / 2, C D\}$ in Figure 1 , we have $1 \leqslant s \leqslant 2$.

Remark. Given a triangle $\Delta R S T$ with $R S=d(R S T)$, to decide whether or not $\Delta R S T \in(\rho-x, \sigma, x+\tau)$ or $(x, \tau, \rho+\sigma-x)$ for some $(\rho, \sigma, \tau)$ where the various angles satisfy the conditions of Theorem 2, bisect $R S$ at $W$ (say). Examine the triangles $\triangle R W T$ and $\triangle W S T$. If one of these is in $(\rho, \sigma, \tau)$, where the angles satisfy the conditions of Theorem 2, then

(i) $2 W T \geqslant R S \Rightarrow \triangle R S T$ is in the corresponding $(\rho-x, \sigma, x+\tau)$,

(ii) $2 W T \leqslant R S \Rightarrow \Delta R S T$ is in the corresponding $(x, \tau, \rho+\sigma-x)$.

Various conditions sufficient for a given triangle $\triangle X Y Z$ to lie in one of the four sets of similarity classes considered can be obtained by elementary calculations using the cosine rule for triangles. For example, given $\Delta X Y Z \in(\alpha, \beta, \gamma)$ with $X Y \geqslant Y Z \geqslant$ $X Z$ and $\alpha \geqslant \beta \geqslant \gamma$, then

(i) if $\cos \gamma \leqslant 3 / 4$, then $\triangle X Y Z \in(\rho, \sigma, \tau)$ satisfies the conditions of Theorem 1;

(ii) if $X Y / Y Z \geqslant 2 / \sqrt{ } 3$ and $\cos \gamma \leqslant \sqrt{ } 3 / 2$, then $\Delta X Y Z \in(\rho, \sigma, \tau)$ and $\Delta X Y Z \in$ $(x, \rho-x, \pi-\rho)$, both satisfying the conditions of Theorem 1;

(iii) if $3 / 4 \geqslant \cos \beta \geqslant \max \{X Z / X Y, X Y / 4 X Z\}$, then $\Delta X Y Z \in(x, \tau, \rho+\sigma-x)$ satisfies the conditions of Theorem 2 . 
Department of Mathematics

University College

Cork, Ireland

1. G. DAHLQUIST \& A. BJORCK, Numerical Methods, translated by N. Anderson, PrenticeHall, Englewood Cliffs, N. J., 1974.

2. R. B. KEARFOTT, "A proof of convergence and an error bound for the method of bisection in $\mathrm{R}^{n}$," Math. Comp., v. 32, 1978, pp. 1147-1153.

3. I. G. ROSENBERG \& F. STENGER, "A lower bound on the angles of triangles constructed by bisecting the longest side," Math. Comp., v. 29, 1975, pp. 390-395.

4. M. STYNES, "An algorithm for numerical calculation of topological degree," Applicable Anal. (To appear.)

5. M. STYNES, "On the construction of sufficient refinements for computation of topological degree," Numer. Math. (Submitted.) 